\title{
Magnetic Induced Heating of Nanoparticle Solutions
}

\author{
M. Brown, ${ }^{1}$ K. Coopersmith, ${ }^{1}$ S. Fulmer, ${ }^{1}$ H. Sessions, Jr., ${ }^{1}$ M. Ali ${ }^{2}$ and S. Hunyadi Murph ${ }^{1,3}$ \\ ${ }^{1}$ National Security Directorate, Savannah River National Laboratory, Aiken, SC 29803, USA \\ ${ }^{2}$ College of Engineering and Computing, University of South Carolina, SC 29208, USA \\ ${ }^{3}$ Department of Physics and Astronomy, The University of Georgia, Athens, GA 30602, USA
}

\begin{abstract}
Magnetic induced heating of nanoparticles (NP) provides a useful advantage for many energy transfer applications. This study aims to gain an understanding of the key parameters responsible for maximizing the energy transfer leading to nanoparticle heating through the use of simulations and experimental results. It was found that magnetic field strength, NP concentration, NP composition, and coil size can be controlled to generate accurate temperature profiles in NP aqueous solutions.
\end{abstract}

\section{INTRODUCTION}

An alternating magnetic field (AMF) has the potential to heat nanoparticles by utilizing the physical and magnetic properties of the nanoparticles. Magnetic induced heating of nanoparticles is a non-contact type of heating that has many benefits and potential applications compared with different forms of contact heating like resistive heaters. The most notable applications are biomedical applications like in vivo sensing, separation of biomolecules, controlled drug release, and tumor ablation for cancer therapy [1]-[5]. In these applications, nanoparticles are used to facilitate processes that are difficult to monitor or control due to the size and/or heat transfer limitations of current technology.

The process of magnetic induced heating involves applying an alternating magnetic field of known magnitude and frequency to a magnetic material typically through the use of an induction coil. The mechanisms behind nanoparticle heating are due to the hysteresis loss, Néel relaxation loss, and Brownian relaxation loss [1], [4]-[6]. Heat generation due to these mechanisms can be optimized by controlling the magnetic properties of the material, the geometry of the material, and the material environmental conditions.

The beauty of the magnetic induced heating is that MNPs are targeted and the heating can be done remotely and efficiently without heating unwanted objects. [5] The magnetic field targets the objects of interest and heat is generated quickly and directly due to the mechanisms mentioned earlier. The rate of temperature increase can be controlled by varying the magnitude of the magnetic field which can easily be controlled by changing the current through the coil [3].

Magnetic nanoparticles (MNP) are used because they can easily be functionalized with other materials. Local heating is then achieved due to the targeting ability of the magnetic field in the presence of magnetic material. The MNPs become a receptor of energy from the magnetic field and deliver that energy as heat to the particles or molecules on their surface.

Nanoparticles are typically used instead of bulk material when the intended target is on the nano or micro scale. For example, several studies show that nanoparticles are used in cancer and drug release treatment within the body because nanoparticles can enter the body with no harm and target cancer cells or other affected parts of the body [2], [4], [5].

The target temperature of nanoparticles for biomedical applications is typically around $41{ }^{\circ} \mathrm{C}-46{ }^{\circ} \mathrm{C}$ for the application to be effective and provide the least discomfort or harm for the patient [5]. The research presented in this paper aims to demonstrate that an alternating magnetic field can be used to couple energy to magnetic nanoparticles and deliver heat $\left(\mathrm{T}>50{ }^{\circ} \mathrm{C}\right)$ to nearby particles.

\section{EXPERIMENTAL SETUP}

An AMF was generated using a magnetic hyperthermia system from MSI Automation, Inc. The system has a maximum power of $10 \mathrm{~kW}$ and several coil sizes ranging from 3 to $15 \mathrm{~cm}$. The coil is water-cooled and the frequency of the system is auto-tuned based on the coil inductance and the load coupled to the coil. The percentage of power delivered can be controlled by the user, thus, the magnetic field strength can be manually controlled. The coil used has 6 turns with a turn radius measured at $17.5 \mathrm{~mm}$. The height of the coil was measured to be $47.5 \mathrm{~mm}$. At 80 percent power, the current into the system was measured at 17 amps which corresponds to a maximum magnetic flux density $\left(\mathrm{B}_{\max }\right)$ of $2.7 \mathrm{mT}$ at the center of the coil using Equation 1.

$$
B_{\max }=\mu_{0} n I
$$

where $\mu_{0}$ is the permeability of free space, $n$ is the number of turns per unit length $(N / l)$, and $I$ is the current through the coil. This result is an estimation based on Ampere's Law that assumes an infinite coil length and does not take into account coil geometries [7]. A simulation of the coil in ANSYS Maxwell was done to compare the simulated magnetic field strength to the magnetic field strength calculated from the measured current and finite geometries.

To heat the nanoparticles, MNPs were placed inside of a small vial in de-ionized water. A Neoptix fiber optic temperature probe was placed in the center of the solution for temperature recording. The vial was placed in the center of the coil and the magnetic hyperthermia system was turned on at $80 \%$ power. The temperature rise in the solution with respect to nanoparticle composition and concentration was investigated experimentally while the effects of coil geometries were investigated theoretically using simulations. 


\section{RESULTS}

\section{A. Simulation Studies}

At $80 \%$ power (17 amps) and a $35 \mathrm{~mm}$ diameter coil, the frequency of the magnetic heating system auto-tunes to 425 $\mathrm{Hz}$. Simulated results of a coil with the same geometries, frequency, and applied current are shown in Fig. 1.

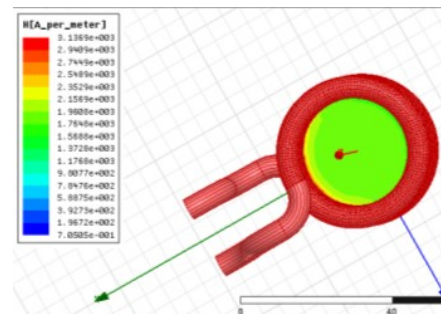

Fig. 1: Magnetic field strength at the center of the coil

The simulation shows that the magnetic field strength at the center of the coil is about $1760 \mathrm{~A} / \mathrm{m}$, which corresponds to a magnetic flux density of $2.2 \mathrm{mT}$ using the relationship $\mathrm{B}=$ $\mu_{0} \mathrm{H}$. Using Equation 2, which was derived from the BiotSavart law [7], the magnetic field at the axial center of the coil with respect to the distance from the center along the length of the coil, $l$, can be calculated.

$$
B=\frac{B_{\max }}{2}\left[\frac{\left(\frac{l}{2}\right)-z}{\sqrt{(z-l / 2)^{2}+r^{2}}}+\frac{\left(\frac{l}{2}\right)+z}{\sqrt{(z+l / 2)^{2}+r^{2}}}\right]
$$

where $B_{\max }$ is the maximum flux density from Equation $1, l$ is the coil length, $r$ is the radius of the coil, and $\mathrm{z}$ is the distance from the center along the length of the coil. Using this equation, the magnetic flux density was determined from the measured current and coil geometries to be $2.17 \mathrm{mT}$ at the center of the coil $(\mathrm{z}=0 \mathrm{~m})$. This confirms the simulated result of $2.2 \mathrm{mT}$.

Looking at Equation 2, the main inhibitor of the magnetic flux density, $B$, reaching its maximum value, $B_{\max }$, is the length of the coil and the radius of the coil. As the radius increases, the flux density decreases. Therefore, it is important to keep the radius of the coil small for a stronger, more focused magnetic field at a constant coil length.

\section{B. Experimental Studies}

Plots for the temperature dependence of the solution on the

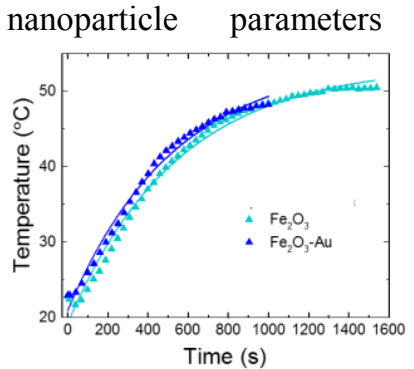

(a)

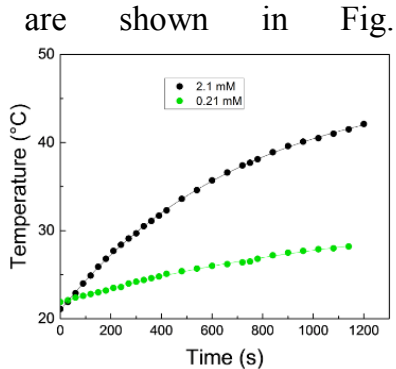

(b)
Fig. 2: Temperature dependence on NP parameters. (a) NP composition (b) NP concentration
The temperature profile dependence on the composition of the nanoparticle for $\mathrm{Fe}_{2} \mathrm{O}_{3}$ and $\mathrm{Fe}_{2} \mathrm{O}_{3}$-Au are shown in Fig. 2a. The presence of gold on the surface of the iron oxide nanoparticle has no positive or negative impact on the initial rate of temperature increase or the final temperature that the solution reaches. The temperature of the solution reaches 50 ${ }^{\circ} \mathrm{C}$ in approximately 20 minutes for both nanoparticle compositions. This is important because this shows that at such small sizes, the heat generated is due to magnetic heating rather than eddy currents within the material since the presence of the gold did not add any additional heat to the solution. This also shows that we can couple the MNPs with other non-magnetic metals without negatively affecting the temperature response of the particles.

The amount of temperature increase as well as the rate of temperature increase is strongly dependent on nanoparticle concentration (Fig. 2b). At $2.1 \mathrm{mM}$ of $\mathrm{Fe}_{2} \mathrm{O}_{3}$, the $\Delta \mathrm{T}$ is $4 \mathrm{x}$ larger than that of the $0.21 \mathrm{mM}$ concentration of $\mathrm{Fe}_{2} \mathrm{O}_{3}$. Each nanoparticle can be considered to be responsible for part of the temperature rise in the solution. Therefore, when there are more nanoparticles to absorb energy from the magnetic field, there will be more heat contributed to the solution. These results are encouraging because parameters like coil size, magnetic field amplitude, and nanoparticle concentration and composition are relatively easy to change so that the heating response can be optimized and controlled.

\section{ACKNOWLEDGEMENT}

Financial support for this work was provided by the Department of Energy (DOE) and the Laboratory Directed Research and Development (LDRD) Strategic Initiative Program at the Savannah River National Laboratory (SRNL).

\section{REFERENCES}

[1] Y. Yamamoto, K. Horiuchi, M. Takeuchi, N. Tanaka, R. Aihara, N. Takeuchi, and S. Fujita. "Size Dependent Study on Magnetic Heating Properties of Superparamagnetic Iron Oxide Nanoparticles Suspension," Journal of Applied Physics, 116, 123906 (2014).

[2] A. L. Glover et al., "Magnetic Heating of Iron Oxide Nanoparticles and Magnetic Micelles for Cancer Therapy," in IEEE Transactions on Magnetics, vol. 49, no. 1, pp. 231-235, Jan. 2013.

[3] S. A. Rovers et al., "Characterization and Magnetic Heating of Commercial Superparamagnetic Iron Oxide Nanoparticles," The Journal of Physical Chemistry, Jul. 2009.

[4] B. C. Chen, C. Y. Ho, L. J. Kao, W. C. Wu, Y. H. Tsai and C. Ma, "Hysteresis Loss-Induced Temperature in Ferromagnetic Nanoparticle," in IEEE Transactions on Magnetics, vol. 50, no. 1, pp. 1-4, Jan. 2014.

[5] S. L. McGill, C. L. Cuylear, N. L. Adolphi, M. Osinski and H. D. C. Smyth, "Magnetically Responsive Nanoparticles for Drug Delivery Applications Using Low Magnetic Field Strengths," in IEEE Transactions on NanoBioscience, vol. 8, no. 1, pp. 33-42, March 2009.

[6] R. Hergt, S. Dutz, R. Müller, M. Zeisberger. "Magnetic Particle Hyperthermia: Nanoparticle Magnetism and Materials Development for Cancer Therapy," Journal of Physics: Condensed Matter, vol. 18, Sept. 2006

[7] S. Liao, P. Dourmashkin, J. W. Belcher. MIT. "Sources of Magnetic Fields," 2004. 